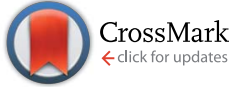

Cite this: RSC Adv., 2017, 7, 11381

Received 30th December 2016 Accepted 10th February 2017

DOI: $10.1039 / c 6 r a 28866 a$

rsc.li/rsc-advances

\section{Highly stable PDMS-PTFPMS/PVDF OSN membranes for hexane recovery during vegetable oil production $\uparrow$}

\begin{abstract}
Xiang Li, ${ }^{a}$ Binglun Chen, ${ }^{a}$ Weibin Cai, ${ }^{a}$ Tao Wang, ${ }^{a}$ Zhen $\mathrm{Wu}^{\mathrm{b}}$ and Jiding Li ${ }^{\star a}$
There is a lack of stable and hydrophobic organic solvent nanofiltration (OSN) membranes meaning that their implementation in non-polar solvent nanofiltration remains a challenge, typically in solvent (e.g. hexane) recovery during vegetable oil production (e.g. soybean oil/hexane). Considering this, novel trifluoropropylmethylsiloxane dimethylsiloxane (PDMS-PTFPMS)/polyvinylidene fluoride (PVDF) membranes, with both high hexane permeability of PDMS and excellent hexane stability of PTFPMS, were successfully developed to recover hexane via OSN. Their microstructure and surface properties were characterized by SEM, FTIR, DSC, TGA, XPS and contact angle measurements. We explored the effects of the ratio of the PTFPMS segment in polymer chain and polymer viscosity on membrane performance. With regards to the F50-M membrane, we also conducted a comprehensive study on the OSN performance of the F50-M membrane under different operational conditions. The oil rejection was above $95 \%$ with stable hexane permeability of $3.06 \mathrm{~kg}^{-1} \mu \mathrm{m} \mathrm{m}^{-2} \mathrm{~h}^{-1}$ bar $^{-1}$ over a 32-day period. Experimental results confirmed its ability to recover hexane under various operational conditions, showing high oil rejection and excellent long-term operational stability. We attributed its outstanding performance to its unique microstructure and surface properties due to the fluorine-containing PTFPMS segment. This study indicates that fluoropolymer membranes are promising candidates in OSN processes, offering a wider choice of membrane materials and application fields.
\end{abstract}

\section{Introduction}

Solvent recovery during vegetable oil production (e.g. soybean oil) is usually performed in aggressive non-polar organic solvents, for instance hexane. Crude miscella is a mixture of 70$75 \mathrm{wt} \%$ hexane and $25-30 \mathrm{wt} \%$ soybean oil after soybean oil extraction. Obviously, concentrating the oil from a concentration of $25 \mathrm{wt} \%$ to $50 \mathrm{wt} \%$ or higher requires an extremely high level of energy consumption, as a result of thermal evaporation. However, it is expected to fulfil the same concentrating goal consuming less energy via organic solvent nanofiltration (OSN). ${ }^{1}$

Despite the availability of large amounts of OSN membranes, most of them are used in separating polar organic solvent mixtures. ${ }^{2,3}$ There are few membranes which can be utilized to separate non-polar organic solvent mixtures, for instance polysulfone membranes. ${ }^{4}$ However, it should be noted that they lack durability in these solvents. In terms of durability, ceramic

${ }^{a}$ The State Key Laboratory of Chemical Engineering, Department of Chemical Engineering, Tsinghua University, Beijing 100084, China. E-mail: lijiding@mail. tsinghua.edu.cn

${ }^{b}$ Ordos Redbud Innovation Institute, Ordos 017000, China

$\dagger$ Electronic supplementary information (ESI) available. See DOI: $10.1039 / \mathrm{c} 6 \mathrm{ra} 28866 \mathrm{a}$ membranes are more suitable with their higher stability towards non-polar organic solvents. Yet hydrophobic ceramic membranes are difficult to produce with separation properties in the nanofiltration range, not to mention they are brittle and costly. ${ }^{5}$ So, the search for efficient and cost-effective non-polar OSN membranes continues. The implementation of OSN in non-polar organic solvents remains a challenge, especially in aliphatic solvent separations. ${ }^{1}$ According to previous publications, ${ }^{3,6,7}$ the key is to fabricate a hydrophobic membrane surface benefiting non-polar solvent permeation, and at the same time to avoid damaging the membrane following longterm operation in non-polar organic solvents.

PDMS - one of the intrinsically hydrophobic materials - can be cross-linked to form a hydrophobic membrane, and can be used in hexane recovery during the production of vegetable oil. ${ }^{8}$ However, it is not suitable for long-term operation due to excessive swelling. ${ }^{9-11}$ Despite efforts made to solve this issue, including incorporating nanoparticles into the membrane, ${ }^{\mathbf{1 0}}$ and utilizing improved cross-linking, ${ }^{12}$ inadequate stability and low solvent permeation still limits its further use in industry. On the other hand, the enhancement of both the permeability of non-polar solvents and molecular weight cut off (MWCO) has been confirmed via chemical modification based on ceramic porous substrates. ${ }^{5,7,13-16}$ Ana F. M. Pinheiro et al. reported that grafting polydimethylsiloxane (PDMS) oligomer onto the 


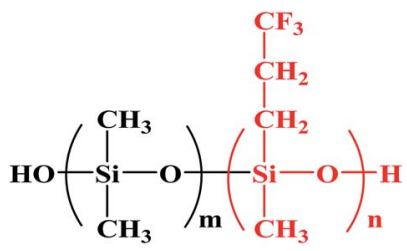

Fig. 1 General structure of silanol terminated trifluoropropylmethylsiloxane dimethylsiloxane copolymer (PDMS-PTFPMS), which is PDMS when $n=0, m=1$; and PTFPMS when $m=0, n=1$.

alumina porous substrate showed high hexane permeability of $4.8 \mathrm{~L} \mathrm{~m}^{-2} \mathrm{~h}^{-1}$ bar $^{-1}$ and a low MWCO value of $500 \mathrm{Da}^{5}$ However, this cumbersome procedure blocked its large-scale production. Herein, we propose to utilize new copolymer, which can combine the polytrifluoropropylmethylsiloxane (PTFPMS) of intrinsic resistance to non-polar solvents with PDMS, to prepare highly stable OSN membranes with enough hexane permeability.

The polytrifluoropropylmethylsiloxane (PTFPMS) is a fluoropolymer rubber composed of repeating methyl(3,3,3trifluoropropyl) siloxane units shown in Fig. 1. It possesses good low-temperature stability-a glass transition temperature of $-74{ }^{\circ} \mathrm{C}$ and strong hydrophobicity, a broad chemical resistance and good weatherability. ${ }^{17}$ PTFPMS has outstanding resistance to hydrocarbon solvent or oil. ${ }^{18}$ On the other hand, the previous report proved that the clever introduction of fluoro-containing groups could enhance non-polar solvent permeation across the membrane. ${ }^{7}$ PTFPMS membranes have been applied in pervaporation, ${ }^{\mathbf{1 7 , 1 9 , 2 0}}$ and gas separation. ${ }^{\mathbf{2 1 - 2 3}}$ However, no reported publication focuses on the feasibility of PTFPMS in OSN processes. It can be expected that PDMS-PTFPMS membranes may effectively combine the advantages of the high hexane permeability of PDMS and the excellent hexane stability of PTFPMS. Moreover, we believe that the appropriate ratio of PTFPMS segment in the polymer chain is critical to realize high non-polar solvent (e.g. hexane) permeation with long-term durability in industrial processes.

In this study, the effects of the ratio of PTFPMS segment and polymer viscosity on membrane physical/chemical structures and OSN performance were investigated to verify the effectiveness in the enhancement of hexane permeability and oil rejection. Furthermore, a comprehensive study on recovering hexane from the soybean oil/hexane mixture was conducted under different operational parameters (including oil concentration, operational temperature and time) to confirm the feasibility of the application of PDMS-PTFPMS/PVDF OSN membrane during vegetable oil production.

\section{Experimental}

\subsection{Materials}

The silanol polydimethylsiloxane (Dingye Inc.) and silanol terminated trifluoropropylmethyl-siloxane dimethylsiloxane copolymers (Russia) were utilized and their specifications are shown in Table 1. Tetramethoxysilane (TMOS, 98\%, Aladdin)
Table 1 Specifications of PDMS-PTFPMS copolymers for membrane preparation

\begin{tabular}{|c|c|c|c|}
\hline & Abbreviation & $\begin{array}{l}n /(m+n) \\
(\%)\end{array}$ & $\begin{array}{l}\text { Viscosity } \\
\text { (Pa s) }\end{array}$ \\
\hline \multirow{7}{*}{$\begin{array}{l}\text { Silanol polydimethylsiloxane } \\
\text { Silanol terminated } \\
\text { trifluoropropylmethyl- } \\
\text { siloxane dimethylsiloxane } \\
\text { copolymers }\end{array}$} & PDMS & 0 & 10 \\
\hline & F15 & 15 & 11 \\
\hline & F25 & 25 & 12 \\
\hline & F100 & 100 & 13 \\
\hline & F50-L & 50 & 1 \\
\hline & F50-M & 50 & 5 \\
\hline & F50-H & 50 & 23 \\
\hline
\end{tabular}

was used as the cross-linking agent and dibutyl tin diacetate (DBTA, Alfa-Aesar) as the catalyst. Butanone (AR) and $n$-hexane (AR) were purchased from Beijing Chemical Works (Beijing, P. R. China). All reagents were utilized as received. To investigate membrane separation performance, a model solution composed of a certain amount of soybean oil (Third-grade, Luhua Inc.) and $n$-hexane was prepared.

\subsection{Preparation of PDMS-PTFPMS/PVDF composite membranes}

PDMS-PTFPMS membranes were prepared via a facile solutioncasting technology. The PDMS-PTFPMS copolymer is added into butanone to form the $25 \mathrm{wt} \%$ homogeneous solution at $10{ }^{\circ} \mathrm{C}$. And the following is the addition of crosslinking agent and catalyst with mass proportion of $100: 10: 3.5$ (polymer: TMOS : DBTA). The resulting solution was further stirred and cross-linked to obtain enough viscosity for casting (see in Table $\mathrm{S} 1 \dagger)$. Then the pre-crosslinking solution was casted rapidly on a PVDF ultrafiltration support previously prepared using nonsolvent-induced phase separation (NIPs) (see ESI $\dagger$ ). ${ }^{24}$ The assynthesized membrane was dried for 8-10 h at room temperature to remove most of the butanone. Further annealing at $100{ }^{\circ} \mathrm{C}$ for $10 \mathrm{~h}$ was required before undergoing various characterizations and organic solvent nanofiltration experiments. The resulting cross-linked PDMS-PTFPMS/PVDF membranes are denoted as PDMS, F15, F25, F100, F50-L, F50-M and F50-H membranes for ease of further discussion as below.

Some characterizations, including differential scanning calorimetry (DSC), thermal gravimetric (TG) and determination of gel content and swelling degree, required dense cross-linked PDMS-PTFPMS films. We poured the pre-crosslinking casting solution onto the PTFE pan. Following this, the same heattreatment procedure was also utilized. The resulting membranes were denoted as PDMS, F15, F25, F100, F50-L, F50$\mathrm{M}$ and $\mathrm{F} 50-\mathrm{H}$ films for ease of further discussion as below.

\subsection{Membrane characterization}

Fourier Transform-Infrared (FTIR) spectra were recorded on a Thermo Scientific* Nicolet* 8700 spectrometer, with samples mounted on a zinc-selenium/diamond plate. The spectra were recorded at a resolution of $4 \mathrm{~cm}^{-1}$ from an average of 32 scans. SEM images were obtained using a JEOL JSM-7401F field- 
emission instrument. The samples were cryogenically fractured in liquid nitrogen and then coated with gold under a vacuum. XPS spectra of cross-linked PDMS-PTFPMS/PVDF composite membranes were collected with a monochromatic $\mathrm{Al} \mathrm{K} \alpha$ radiation source, with a take-off angle of $45^{\circ}$, over a range of 0 $1200 \mathrm{eV}$. The detection depth was approximately $2 \mathrm{~nm}$. The thermal stability and degradation behavior of cross-linked films was determined using a TG analyzer under $\mathrm{N}_{2}$ atmosphere at a heating rate of $10^{\circ} \mathrm{C} \mathrm{min}{ }^{-1}$ from $30^{\circ} \mathrm{C}$ to $800^{\circ} \mathrm{C}$. Additionally, the glass transition temperature $\left(T_{\mathrm{g}}\right)$ was measured by DSC on Seiko DSC6200. $\mathrm{N}_{2}$ atmosphere from $-140{ }^{\circ} \mathrm{C}$ to $20{ }^{\circ} \mathrm{C}$ was adopted and the cooling and heating rate was held constant at $10{ }^{\circ} \mathrm{C} \mathrm{min}{ }^{-1}$. Static contact angle measurements were conducted on the surface of cross-linked PDMS-PTFPMS/PVDF membranes using a Dataphysics OCA20 (Dataphysics Instruments $\mathrm{GmbH}$, German) at room temperature. The test liquid was deionized water. Five contact angles at different locations on the same surface were recorded and averaged for reliability.

\subsection{Determination of gel content and swelling degree}

Gel content was measured through a facile organic solvent extraction method. The cross-linked film sample was immersed in pure butanone and then was removed, dried in the oven and finally weighed after refluxing for $24 \mathrm{~h}$. The gel content $(G)$ was calculated as follows:

$$
G=\frac{m_{0}-m_{\mathrm{G}}}{m_{0}} \times 100 \%
$$

where, $m_{0}$ is the initial weight of the dry membrane sample and $m_{\mathrm{G}}$ the residual weight of the film sample after butanone extraction.

The swelling degree of a dense cross-linked film was acquired by swelling experiments using a pre-determined solution. The pre-weighed sample was immersed in a $26 \mathrm{wt} \%$ soybean oil/hexane solution at room temperature. The sample reached a state of equilibrium after three days, and was quickly removed, wiped with filter paper and weighed. The swelling degree $(S)$ was calculated by:

$$
S=\frac{m_{\mathrm{S}}-m_{0}}{m_{0}} \times 100 \%
$$

where, $m_{0}$ is the weight of the dry film sample and $m_{\mathrm{S}}$ the weight of the swollen film sample.

\subsection{Soybean oil/hexane separation test}

Organic solvent nanofiltration or soybean oil/hexane separation property of cross-linked PDMS-PTFPMS/PVDF composite membranes was determined in a custom-made apparatus with two cross-flow cells shown in Fig. 2. The reservoir was charged with a model solution at a pre-set oil concentration. The applied pressure was adjusted using the back pressure regulator (BPR), and the operating temperature was set and held. Permeate flux $J\left(\mathrm{~kg} \mathrm{~m}^{-2} \mathrm{~h}^{-1}\right)$ was determined by measuring the permeate weight accumulated during the operation period at steady-state conditions and was calculated from:

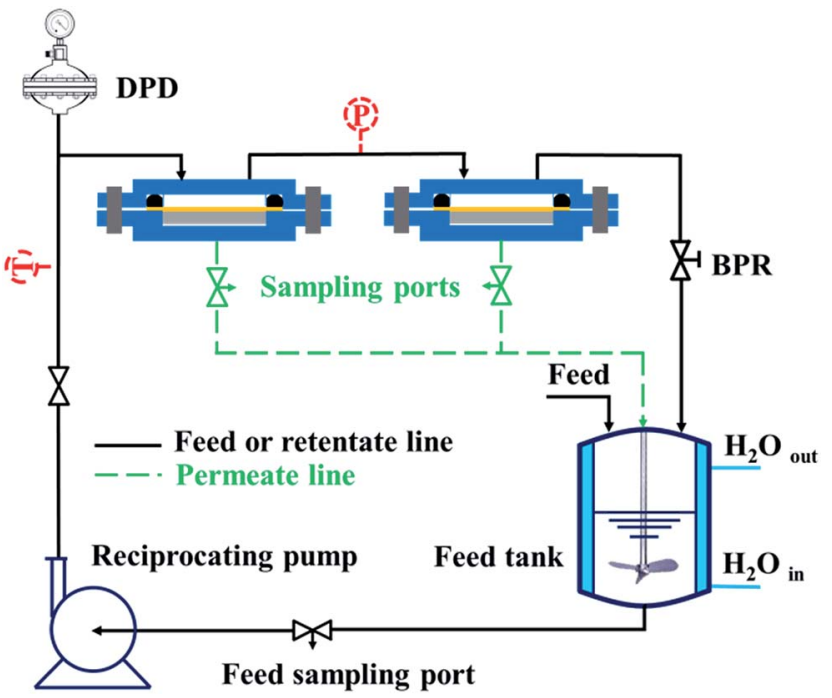

Fig. 2 Schematic representation of the double cells cross-flow rig used in this study. Legend: P - pressure gauge; T - thermocouple; BPR - back pressure regulator; DPD - diaphragm pulsed damper.

$$
J=\frac{W}{A \times t}
$$

where, $W(\mathrm{~kg})$ is the total mass of permeate collected under transmembrane pressure $p(\mathrm{MPa})$ in a time scale $t(\mathrm{~h})$ and $A\left(\mathrm{~m}^{2}\right)$ is the effective membrane area.

$$
R=\left(1-\frac{C_{\mathrm{p}}}{C_{\mathrm{f}}}\right) \times 100 \%
$$

where, $C_{\mathrm{p}}$ and $C_{\mathrm{f}}$ is the solute concentration in the feed and in the permeate, respectively. Concentrations of soybean oil in feed and permeate samples were analyzed using an Abbemat WR refractometer (Anton Paar GmbH, Austria). ${ }^{25}$

\section{Results and discussion}

\subsection{Membrane characterization and analysis}

3.1.1 SEM. Fig. 3 shows SEM images of PDMS, F15, F25 and F100 membranes. These membranes are composed of the porous PVDF substrate and cross-linked PDMS-PTFPMS separation layers, which are confirmed by FTIR (see Fig. S1†). Despite the ratio of PTFPMS segment in the polymer chain, these membranes are dense without defect or patterned morphology on both surface and cross-section. The result indicates that any possible phase separation caused by the introduction of various ratios of PTFPMS segment does not occur. We also determine that the thickness of the separation layer is in the range of 6-8 $\mu \mathrm{m}$. A similar conclusion is obtained from Fig. 3. F50 membranes, formed from various viscosities of F50 polymers, also have a smooth and dense morphology. Thus the introduction of PTFPMS segment cannot bring about any uncertain phase separation. The thickness of F50-L, F50-M and F50- $\mathrm{H}$ membranes is around $7 \mu \mathrm{m}, 15 \mu \mathrm{m}$ and $2 \mu \mathrm{m}$, respectively (Fig. 4). In summary, homogeneous and free-defect PDMS-PTFPMS/PVDF membranes can be acquired from these novel polymers. 


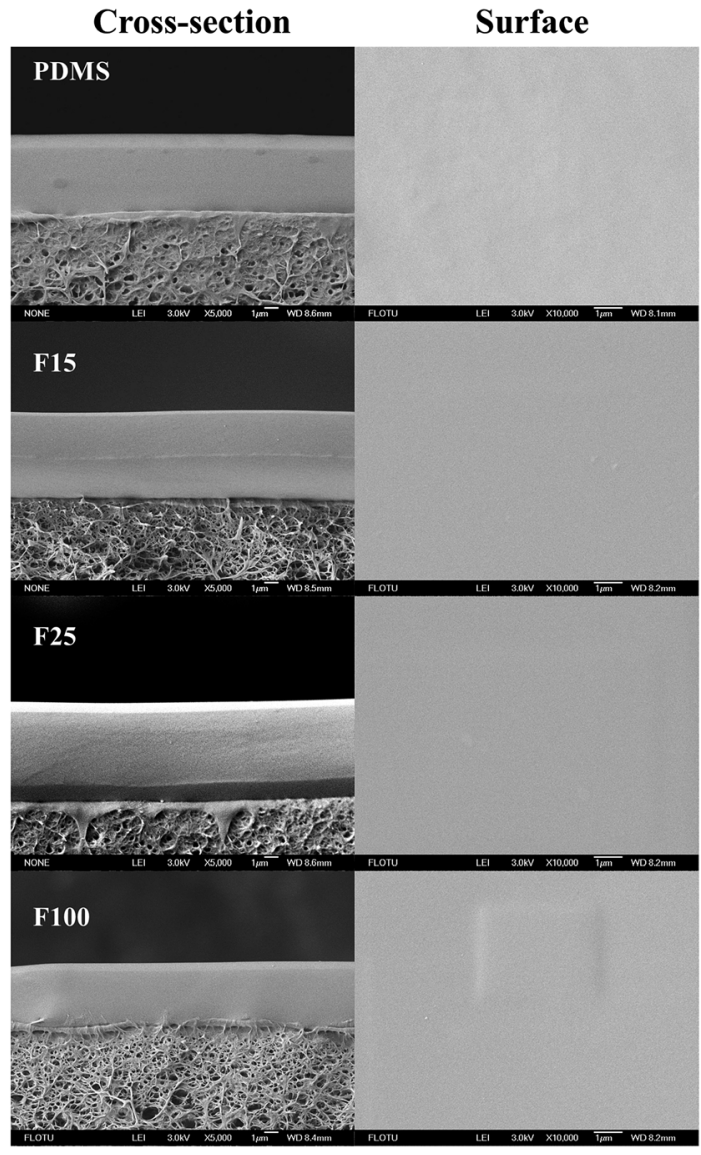

Fig. 3 SEM images of PDMS, F15, F25 and F100 membranes.

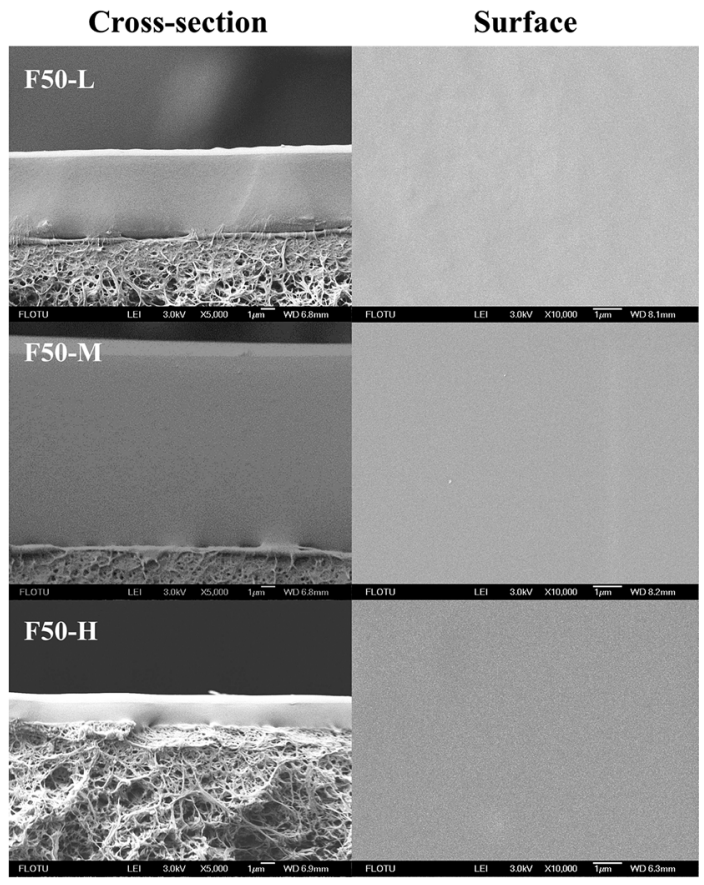

Fig. 4 SEM images of F50-L, F50-M and F50-H membranes.
3.1.2 XPS and contact angle analysis. The surface elemental composition of PDMS-PTFPMS/PVDF composite membranes is determined in Table 2. Increasing the ratio of PTFPMS segment can firstly raise surface F content from PDMS (0) to F15 (14.86 at\%), then from F25 (16.96 at\%) to F100 (27.66 at\%). Also, a similar result is acquired when polymer viscosity increases. Surface F content can be described as F50-L (11.12 at\%) < F50-M (21.60 at\%) < F50-H (37.00 at\%). So surface F content is altered by changing the polymer viscosity or the ratio of PTFPMS segment in polymer chains. In this way, we expected that the affinity of selective layers towards the solvent/solute is controlled, aimed at enhancing the membrane stability and permeability.,26-28

Fig. 5 depicts the contact angle of PDMS-PTFPMS/PVDF composite membranes using DI water as the test liquid. The contact angle is $115.0^{\circ}$ for PDMS membrane, and it initially increases with the growing ratio of PTFPMS segment and then lowers to $118.4^{\circ}$ for F100 membrane. We can see that F15 membranes are at an angle of $119.0^{\circ}$ and F25 at $120.0^{\circ}$. This initial increase is due to the hydrophobicity of the trifluoropropyl group. Yet, the slight decrease in contact angle from F25 membrane to F100 membrane should be attributed to the low cross-linking degree of F100. On the other hand, despite the cross-linking degree, largely increasing surface F content of a membrane can raise its contact angle. This is confirmed from the contact angle of F50 membranes as shown in Fig. 5.

Table 2 Surface elemental composition of cross-linked PDMSPTFPMS/PVDF composite membranes from XPS spectra (in at\%)

\begin{tabular}{lllll}
\hline Samples & C (\%) & O (\%) & Si (\%) & F (\%) \\
\hline PDMS & 49.72 & 25.17 & 25.11 & 0 \\
F15 & 45.12 & 20.80 & 19.22 & 14.86 \\
F25 & 44.40 & 19.67 & 18.97 & 16.96 \\
F100 & 41.60 & 18.20 & 12.54 & 27.66 \\
F50-L & 48.61 & 22.40 & 17.88 & 11.12 \\
F50-M & 43.81 & 18.98 & 15.61 & 21.60 \\
F50-H & 39.43 & 12.61 & 10.96 & 37.00
\end{tabular}

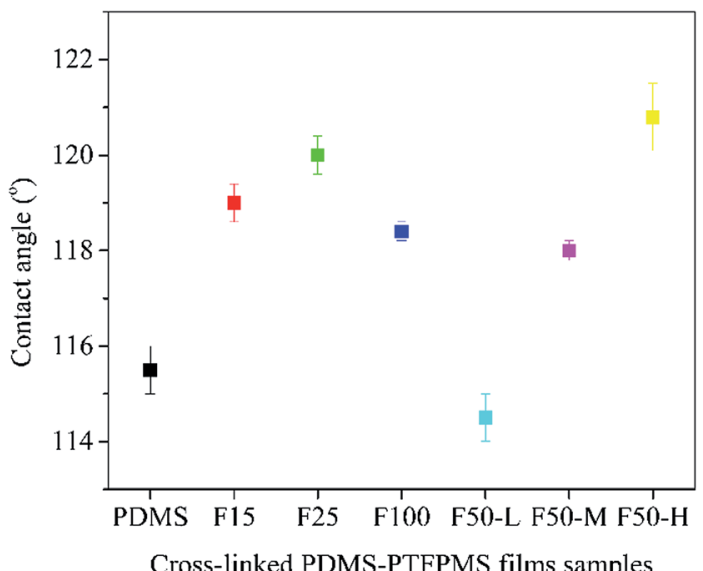

Fig. 5 The contact angle of PDMS-PTFPMS/PVDF composite membranes. 


\subsection{Gel content and swelling degree}

The gel content of cross-linked PDMS-PTFPMS film samples is determined via butanone extraction as is shown in Table 3, reflecting their degree of cross-linking. There is similar gel content for PDMS, F15, F25 and F50-M, showing a value of around $94 \mathrm{wt} \%$. This is consistent to their thermal stability or degradation behaviors (see Fig. S2 and S3 $\dagger$ ). F100, F50-L and F50-H have comparatively lower gel content, indicating lower cross-linking degree and then more free unreacted hydroxyl groups. Resulting transport characteristics of small molecules (e.g. hexane and soybean oil) is affected in the confined permeation channel with various compositions. $^{29,30}$

The swelling degree of cross-linked PDMS-PTFPMS film samples is measured in the model solution shown in Fig. 6 . Swelling degree is highest for the PDMS film and suffers a sharp decline of $82.9 \%$ for the F100 film, showing values of $197.1 \%, 139.6 \%, 86.6 \%$ and $33.8 \%$ for PDMS, F15, F25 and F100, respectively. Also, increasing the viscosity of F50 polymer can decrease swelling degree of corresponding films. The resulting swelling degree is $49.6 \%, 39.2 \%$ and $15.2 \%$ for F50-L, F50-M and F50-H, respectively. Combined with surface elemental analysis, the introduction of PTFPMS segment can inhibit film swelling in hexane-based solution, and then reduce the available pathway to permeate hexane molecules.

Table 3 Gel content of cross-linked PDMS-PTFPMS film samples

\begin{tabular}{lr}
\hline Samples & Gel content \\
\hline PDMS & $94.5 \pm 0.2$ \\
F15 & $93.6 \pm 0.2$ \\
F25 & $93.6 \pm 1.0$ \\
F100 & $59.5 \pm 1.2$ \\
F50-L & $72.6 \pm 2.2$ \\
F50-M & $93.3 \pm 1.0$ \\
F50-H & $84.0 \pm 0.2$
\end{tabular}

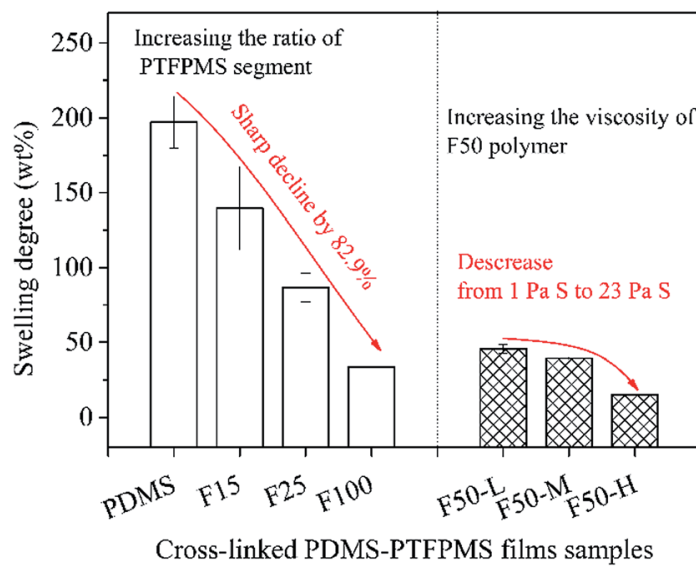

Fig. 6 Swelling degree of cross-linked PDMS-PTFPMS film samples in $26 \pm 1 \mathrm{wt} \%$ soybean oil/hexane (open) or $7 \pm 1 \mathrm{wt} \%$ soybean oil/hexane (pattern)

\subsection{Soybean oil/hexane separation performance}

3.3.1 The effect of the ratio of PTFPMS segment. Fig. 7a describes the effect of the ratio of PTFPMS segment on crosslinked PDMS-PTFPMS/PVDF OSN membrane performance. The good linearity between permeate flux and applied pressure is related to no compaction of cross-linked membranes. ${ }^{8}$ Unexpectedly, permeate flux of the PDMS membrane is $10.5 \mathrm{~kg}$ $\mathrm{m}^{-2} \mathrm{~h}^{-1}$ lower than the $\mathrm{F} 15$ membrane at $2.4 \mathrm{MPa}$. The resulting enhancement of hexane permeation is attributed to the introduction of $\mathrm{F}$ element from PTFPMS segment. ${ }^{7}$ Specifically, more hydrophobic surface of F15 membrane enhances the adsorption of non-polar hexane and onto its surface and hexane diffusion through the membrane. In addition, there is less concentration polarization using F15 membrane than PDMS membrane under same testing conditions. We will now focus further on F15, F25 and F100 membranes. The continuous reduction of permeate flux occurs when the ratio of PTFPMS segment increases, showing values of $15.84 \mathrm{~kg} \mathrm{~m}^{-2} \mathrm{~h}^{-1}, 8.28 \mathrm{~kg}$ $\mathrm{m}^{-2} \mathrm{~h}^{-1}, 1.60 \mathrm{~kg} \mathrm{~m}^{-2} \mathrm{~h}^{-1}$ for F15, F25 and F100 membranes at 2.4 $\mathrm{MPa}$, respectively. According to forgoing analysis in Section 3.1 and 3.2, the reasons for this are as follows: high $\mathrm{F}$ content in the membrane surface and matrix results in less adsorption of hexane in the membrane surface and faster diffusion of hexane through the membrane matrix (Table $\mathrm{S} 3 \dagger$ ); the available permeate channel between polymer chains becomes narrower, which is consistent with the change in swelling degree of these cross-linked films.

Next, rejection curves of soybean oil are utilized to investigate the soybean oil/hexane separation property of cross-linked PDMS-PTFPMS/PVDF composite membranes shown in Fig. 7b. When increasing the ratio of PTFPMS segment, oil rejection is enhanced under the applied pressure of 1.2-2.4 MPa. This is also attributed to narrower permeate channels tending to inhibit the diffusion of large solute molecule (soybean oil). This is also confirmed from RB rejection as shown Table S4. $\uparrow$ Yet oil rejection of the PDMS membrane is $90.0 \%$ higher than the F15 membrane at $0.8 \mathrm{MPa}$. This result indicates that a small amount of trifluoropropyl group (e.g. F15) is also able to raise the sorption of soybean oil, due to its weak polarity. ${ }^{31,32}$

3.3.2 The effect of polymer viscosity. Considering the physical-chemical stability of cross-linked PDMS-PTFPMS membranes (see in Table S2 $\dagger$ ), the F50/PVDF composite membrane was selected for further study on the effect of polymer viscosity on OSN performance as shown in Fig. 8. Fig. 8a and $\mathrm{b}$ show that the F50-M membrane has the best permeability

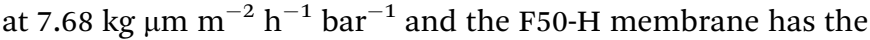
highest oil rejection at $98.9 \%$ at an applied pressure of $2.4 \mathrm{MPa}$. This result differs from that of previous PDMS membranes formed from 5-100 $\mathrm{Pa} \mathrm{s}$ by our group. ${ }^{25,33}$ Previous PDMS membranes only consider polymer viscosity because of its intrinsic dissolution in hexane. Yet F50 polymers cannot dissolve in hexane. Consequently, the available permeation channel is just around the PDMS polymer chain. As a result, longer PDMS chain and less free hydroxyl group stemming from inadequate cross-linking can raise hexane diffusion with the reduction of oil rejection. On the other hand, F50 polymer chain 

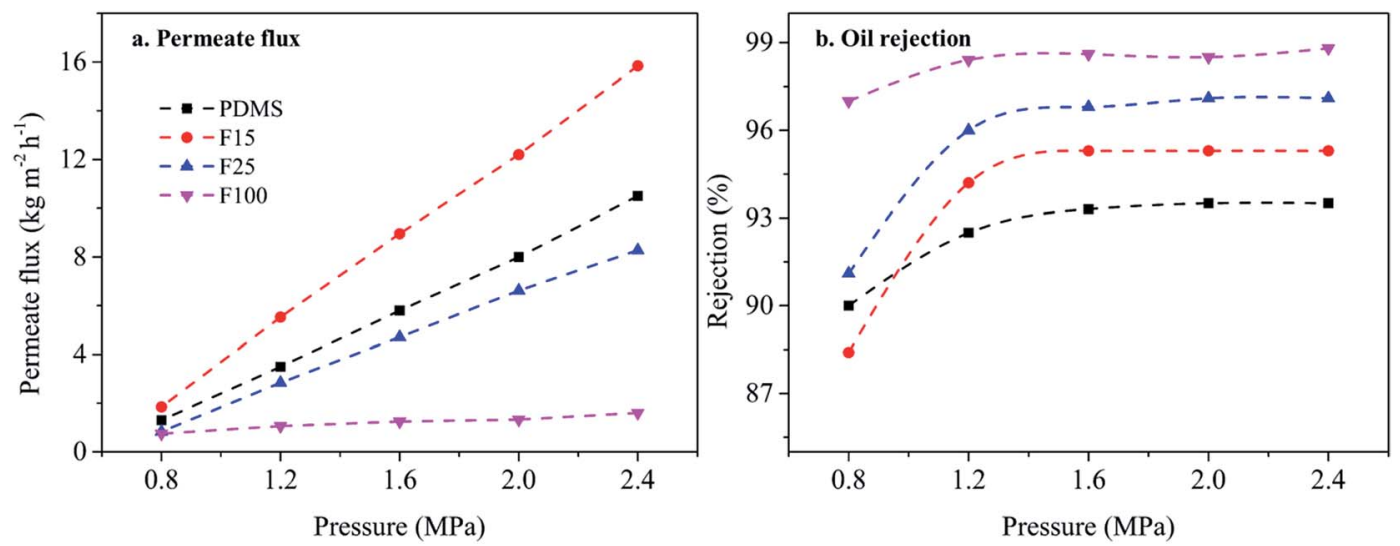

Fig. 7 The effect of the ratio of PTFPMS segment on permeate flux and oil rejection ( $26 \pm 1 \mathrm{wt} \%$ soybean oil/hexane, $25 \pm 1{ }^{\circ} \mathrm{C}$ ).
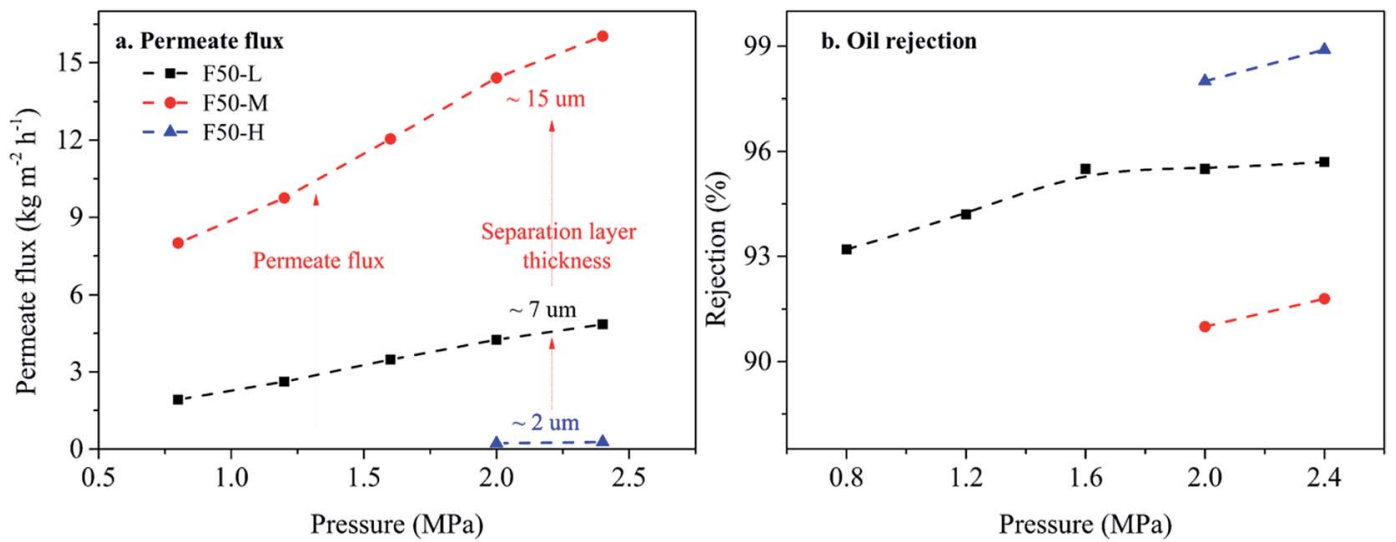

Fig. 8 The effect of polymer viscosity on permeate flux and oil rejection $\left(7 \pm 1\right.$ wt $\%$ soybean oil/hexane, $\left.25 \pm 1^{\circ} \mathrm{C}\right)$.

with longer PDMS chain also has a higher $\mathrm{F}$ content on the membrane surface, which is confirmed in Table 2. This causes less swelling of membranes, and then inhibits hexane diffusion with the enhancement of oil rejection. Accordingly, F50-M membrane is optimal when balancing the intrinsically insolubility in hexane derived from the PTFPMS segment and high permeability of hexane from the PDMS segment.

\subsubsection{Hexane recovery from soybean oil/hexane mixture}

3.3.3.1 The effect of feed oil concentration. The F50-M membrane is the best selection for further study, due to excellent solvent resistance (see Table S2 $\dagger$ ) and high solvent permeability. Fig. 9 describes the effect of feed oil concentration on membrane performance. Permeate flux at feed oil concentration of $14 \mathrm{wt} \%$ and $2.4 \mathrm{MPa}$ is $6.87 \mathrm{~kg} \mathrm{~m}^{-2} \mathrm{~h}^{-1}$ and it linearly
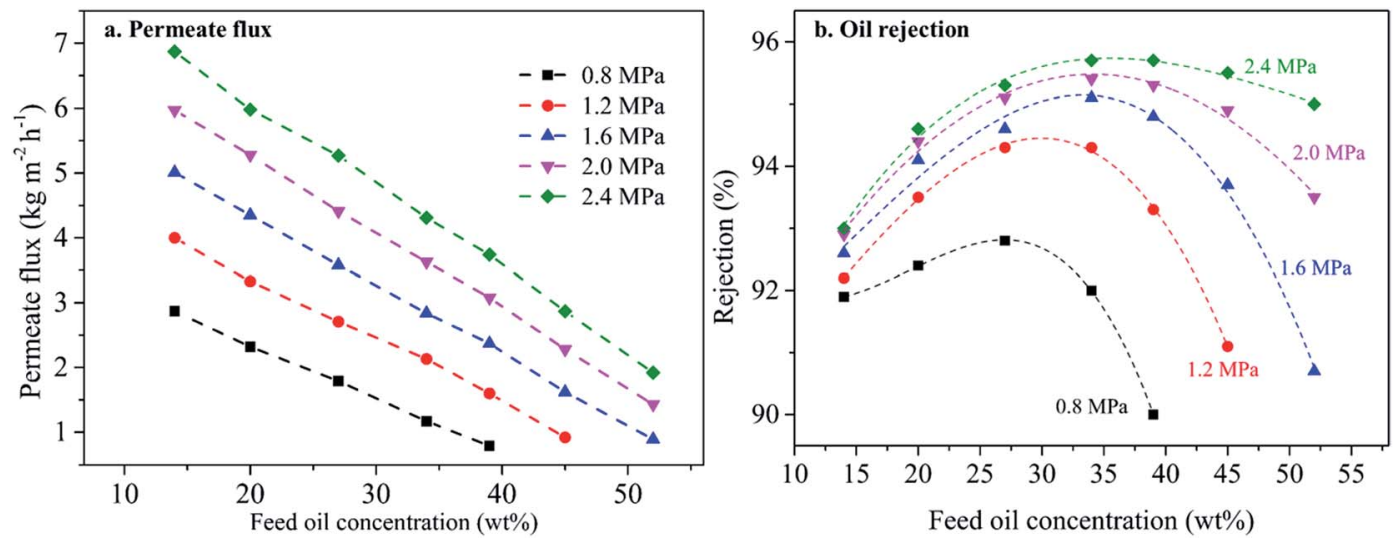

Fig. 9 The effect of feed oil concentration on membrane performance for the F50/PVDF membrane at $25^{\circ} \mathrm{C}$. 

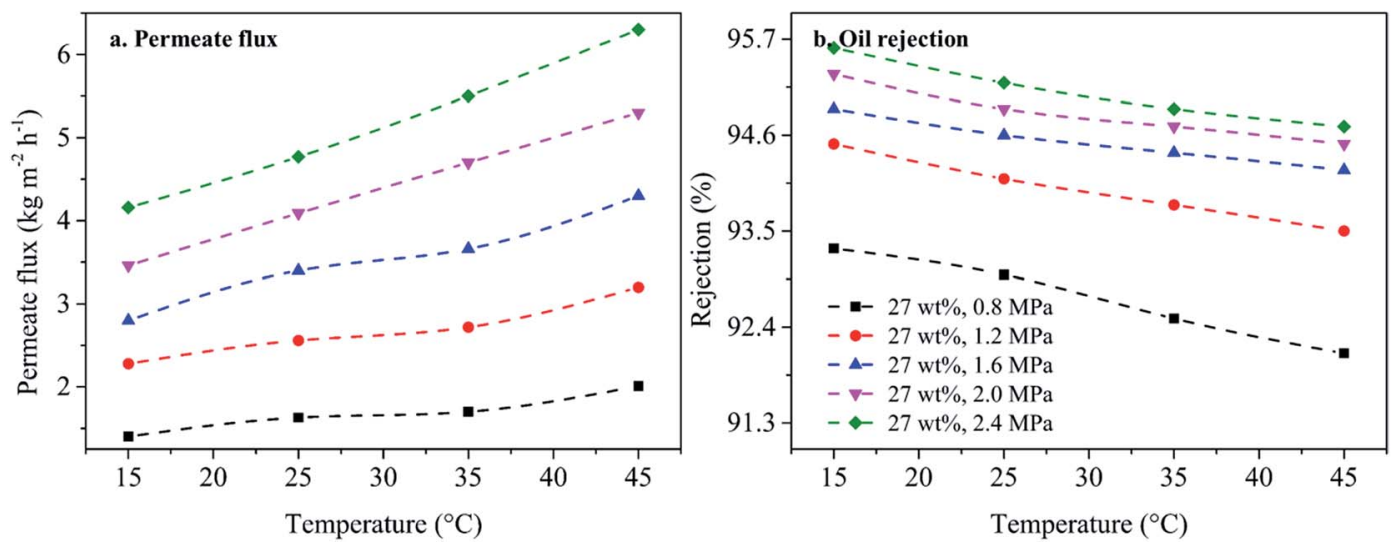

Fig. 10 The effect of operational temperature on membrane performance for the F50/PVDF membrane.

decreases with increasing feed oil concentration, showing very similar permeate behavior over the whole applied pressure of 0.8-2.4 MPa. According to the van't Hoff equation, ${ }^{1,8}$ we believe that increasing feed oil concentration raises osmotic pressure, and then solvent (hexane) diffusion resistance. Nonetheless, oil rejection still remains above $90.0 \%$ over the whole feed oil concentration. Interestingly, oil rejection curves different from PDMS-based membranes are obtained. ${ }^{8,33}$ These resulting convex curves are caused by lower oil concentration near the membrane surface than that of the bulk feed solution, due to its oleophobicity from the PTFPMS segment. Therefore, fluoropolymer membranes are advantageous over traditional membrane (e.g. PDMS) in performing solvent recovery from the miscella.

3.3.3.2 The effect of operational temperature on membrane performance. Next, the effect of operational temperature on membrane performance is investigated as shown in Fig. 10. Permeate flux at $2.4 \mathrm{MPa}$ is $4.16 \mathrm{~kg} \mathrm{~m}^{-2} \mathrm{~h}^{-1}$, and it slightly increases when operational temperature increases, showing very similar permeate behavior at all levels of applied pressure as shown in Fig. 10a. This is attributed to the increasing permeate pathway of molecules at high operational temperatures. Yet, oil rejection at 2.4 MPa decreases from $95.6 \%\left(15^{\circ} \mathrm{C}\right)$ to $94.7 \%\left(45{ }^{\circ} \mathrm{C}\right)$, showing similar rejection properties at all levels of applied pressure as shown in Fig. 10b. Despite applying differing levels of operational pressure and temperature, oil rejection still remains above $92.0 \%$, showing greater thermal stability than PDMS membranes. ${ }^{33}$

3.3.3.3 Operational stability of the membrane. Based on our initial exploration of membrane stability, PDMS, F15 and F25 membranes tend to peel off after a 2-day testing period, but the F50-M membrane has optimal solvent permeance and stability. Fig. 11 shows the change of the F50 membrane performance over a 32-day testing period. Before the initial 12 days, the membrane undergoes various testing conditions including various feed oil concentrations (7-55 wt\%), operational pressures $(0.8-2.4 \mathrm{MPa})$ and temperature $\left(15-45{ }^{\circ} \mathrm{C}\right)$. The resulting permeation flux or oil rejection stays at a similar level, showing excellent stability. The following 20 days of testing is conducted using $26 \pm 1 \mathrm{wt} \%$ soybean oil/hexane mixture at $2.0 \mathrm{MPa}$ and 25

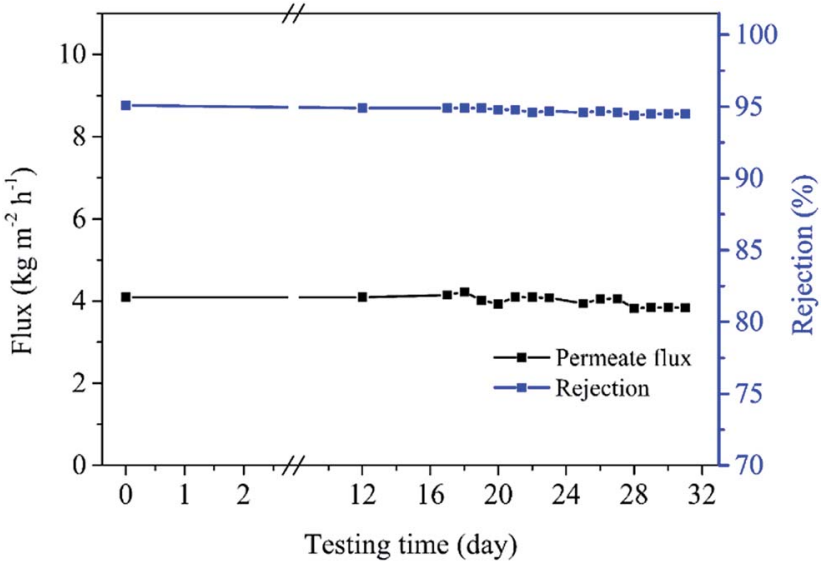

Fig. 11 Permeate flux and oil rejection of the F50 membrane over a 32-day testing period.

$\pm 1{ }^{\circ} \mathrm{C}$. Permeate flux of about $4.09 \mathrm{~kg} \mathrm{~m}^{-2} \mathrm{~h}^{-1}$ (calculated permeability: $3.06 \mu \mathrm{m} \mathrm{kg}^{-1} \mathrm{~m}^{-2} \mathrm{~h}^{-1} \mathrm{bar}^{-1}$ ) and oil rejection of around $95.0 \%$ still remain, showing superior structural integrality to PDMS membranes. Therefore, the F50 membrane, one of the fluoropolymer membranes, is suitable for recovering hexane under various operational conditions.

\section{Conclusion}

Novel PDMS-PTFPMS/PVDF OSN membranes were successfully fabricated for hexane recovery during the soybean oil refining process. Their stability and permeability could be altered via the ratio of PTFPMS segment in polymer chain and polymer viscosity. The F50-M membrane performed the best, effectively combining the advantages of the high hexane permeability of PDMS and excellent hexane stability of PTFPMS. Further comprehensive studies also verified the suitability of the F50-M membrane in recovering hexane from the soybean oil/hexane mixture, showing high hexane permeability of $3.06 \mu \mathrm{m} \mathrm{kg}^{-1}$ $\mathrm{m}^{-2} \mathrm{~h}^{-1} \mathrm{bar}^{-1}$ and oil rejection of around $95 \%$ over a 32-day period under various testing conditions. We attributed its outstanding performance to unique microstructure and surface 
properties of the fluorine-containing PTFPMS segment. The study also indicated that fluoropolymer would widen the selection of OSN membrane materials.

\section{Acknowledgements}

The authors greatly appreciate the financial support of National Natural Science Foundation of China (21576150), Science Foundation of Tsinghua University (20131089399) and the Special funds for technological development research of Research Institutes from National Ministry of Science and Technology (2013EG111129).

\section{References}

1 L. R. Firman, N. A. Ochoa, J. Marchese and C. L. Pagliero, J. Membr. Sci., 2013, 431, 187-196.

2 P. Vandezande, L. E. Gevers and I. F. Vankelecom, Chem. Soc. Rev., 2008, 37, 365-405.

3 P. Marchetti, M. F. Jimenez Solomon, G. Szekely and A. G. Livingston, Chem. Rev., 2014, 114, 10735-10806.

4 M. Saxena, P. Ray, P. S. Singh and A. Bhattacharya, Polym. Bull., 2015, 72, 2157-2169.

5 A. F. M. Pinheiro, D. Hoogendoorn, A. Nijmeijer and L. Winnubst, J. Membr. Sci., 2014, 463, 24-32.

6 M. Amirilargani, M. Sadrzadeh, E. J. R. Sudhölter and L. C. P. M. D. Smet, Chem. Eng. J., 2016, 289, 562-582.

7 M. F. Jimenez Solomon, Y. Bhole and A. G. Livingston, J. Membr. Sci., 2013, 434, 193-203.

8 N. Stafie, D. F. Stamatialis and M. Wessling, J. Membr. Sci., 2004, 228, 103-116.

9 L. E. M. Gevers, I. F. J. Vankelecom and P. A. Jacobs, J. Membr. Sci., 2006, 278, 199-204.

10 L. E. Gevers, I. F. Vankelecom and P. A. Jacobs, Chem. Commun., 2005, 2500-2502.

11 S. Basu, M. Maes, A. Cano-Odena, L. Alaerts, D. E. De Vos and I. F. J. Vankelecom, J. Membr. Sci., 2009, 344, 190-198.

12 S. Aerts, A. Vanhulsel, A. Buekenhoudt, H. Weyten, S. Kuypers, H. Chen, M. Bryjak, L. E. M. Gevers, I. F. J. Vankelecom and P. A. Jacobs, J. Membr. Sci., 2006, 275, 212-219.

13 T. Van Gestel, B. Van Der Bruggen, A. Buekenhoudt, C. Dotremont, J. Luyten, C. Vandecasteele and G. Maes, J. Membr. Sci., 2003, 224, 3-10.
14 C. R. Tanardi, I. F. J. Vankelecom, A. F. M. Pinheiro, K. K. R. Tetala, A. Nijmeijer and L. Winnubst, J. Membr. Sci., 2015, 495, 216-225.

15 S. Rezaei Hosseinabadi, K. Wyns, V. Meynen, R. Carleer, P. Adriaensens, A. Buekenhoudt and B. Van der Bruggen, J. Membr. Sci., 2014, 454, 496-504.

16 N. Gao, M. Li, W. Jing, Y. Fan and N. Xu, J. Membr. Sci., 2011, 375, 276-283.

17 G. Lin, M. Abar and L. M. Vane, Separ Sci Technol, 2013, 48, 523-536.

18 F. Nie, G. He, W. Zhao, J. Ju, Y. Liu and Y. Dai, J. Polym. Res., 2014, 21, 319.

19 X. K. Zhang, Y. Poojari, L. E. Drechsler, C. M. Kuo, J. R. Fried and S. J. Clarson, J. Inorg. Organomet. Polym., 2007, 18, 246252.

20 X. Han, L. Wang, J. Li, X. Zhan, J. Chen and J. Yang, J. Appl. Polym. Sci., 2011, 119, 3413-3421.

21 L. Zhang, G. He, W. Zhao, F. Nie, X. Li and M. Tan, J. Membr. Sci., 2011, 371, 141-147.

22 F. Nie, G. He, W. Zhao, J. Ju and H. Li, CIESC J., 2014, 65, 3019-3025.

23 J. R. Fried and B. Li, in Advanced Materials for Membrane Separations, 2004, ch. 2, pp. 24-38.

24 W. Sun, T. Chen, C. Chen and J. Li, J. Membr. Sci., 2007, 305, 93-102.

25 Y. Sun, W. Cai, X. Piao, J. Li and S. Zhu, Chin. J. Chem. Eng., 2011, 19, 6.

26 M. R. Gómez-Antón, R. M. Masegosa and A. Horta, Polymer, 1987, 28, 2116-2121.

27 G. Cocchi, M. G. De Angelis and F. Doghieri, J. Membr. Sci., 2015, 492, 600-611.

28 S. Postel, C. Schneider and M. Wessling, J. Membr. Sci., 2016, 497, 47-54.

29 N. Stafie, D. F. Stamatialis and M. Wessling, Sep. Purif. Technol., 2005, 45, 220-231.

30 J. P. Robinson, E. S. Tarleton, K. Ebert, C. R. Millington and A. Nijmeijer, Ind. Eng. Chem. Res., 2005, 44, 3238-3248.

31 J. Barrie and D. Machin, J. Macromol. Sci., Part B: Phys., 1969, 3, 645-672.

32 Q. Dai, R. Lebrón-Aguilar, E. Fernández-Sánchez, J. A. García-Domínguez and J. E. Quintanilla-López, J. High Resolut. Chromatogr., 1993, 16, 721-724.

33 W. Cai, Fundamental Research in Solvent Recovery from Oil/ extractant Miscella by Reverse Osmosis Membrane, Ph.D. dissertation, Tsinghua University, Beijing, China, 2013. 